\title{
COLLABORATIVE DOMINANCE: WHEN DOING UNTO OTHERS AS YOU WOULD HAVE THEM DO UNTO YOU IS REASONABLE
}

\author{
Filipe Costa de Souza ${ }^{*}$ and Leandro Chaves Rêgo ${ }^{2}$ \\ Received November 24, 2012 / Accepted August 20, 2013
}

\begin{abstract}
In this article, we analyze how reasonable it is to play according to some Nash equilibria if players have a preference for one of their opponents' strategies. To formalize our argument, we propose the concepts of collaborative dominance and collaborative equilibrium studying some of its properties. First, we prove that if the collaborative equilibrium exists, then it is always efficient, what can be seen as a focal property. Moreover, we argue that one reason that may lead players not to collaborate is that they can focus on security instead of efficiency properties, in which case they would prefer to play according to maximin strategies. This argument allows us to reduce the hall of reasonable equilibria for games where a collaborative equilibrium exists. Finally, we show that two-player zero-sum games do not have collaborative equilibrium, and that if they contain a strategy profile composed only of collaboratively dominated actions, then such profile is a Nash equilibrium of the game.
\end{abstract}

Keywords: Nash equilibrium, collaborative dominance, two-player zero-sum games.

\section{INTRODUCTION}

Even though other authors had previously made isolated contributions to the Theory of Games, many researchers, including Kuhn \& Tucker (1958) and Leonard (1995), considered von Neumann as the father of the Theory of Games because of his initial work in the late 1920 and his further collaboration with Oskar Morgenster that culminated in the publication in 1944 of the classical book Theory of Games and Economic Behavior. Even von Neumann holding this prestigious position in the game theory field, John Nash is the main star in this constellation, largely because of his equilibrium's definition (Nash, 1951). Nowadays, it is almost impossible to talk about game theory without saying Nash's name.

Due to the relevance of this equilibrium concept, many criticisms and even more applications appeared. The first known argument against the Nash equilibrium idea was made by Merrill Flood and Melvin Dresher in an experiment at RAND Corporation. In this experiment, they

\footnotetext{
*Corresponding author

${ }^{1}$ Accounting and Actuarial Science Department, Federal University of Pernambuco, Recife, PE, Brazil.

E-mail: filipe.costas@ufpe.br

${ }^{2}$ Statistics Department, Federal University of Pernambuco, Recife, PE, Brazil. E-mail: leandro@ de.ufpe.br
} 
analyzed how players behaved in a finitely repeated iteration of the prisoners' dilemma (Poundstone, 1992 and Nasar, 1998). On the other hand, the applications started with economic and military subjects and rapidly spread into many other fields such as political science, evolutionary biology, etc. For those readers who seek applications of game theory that make use of the mathematical tractability of operational research, we recommend, for example, the works of Wang \& Parlar (1989), Migdalas (2002) and Machado \& Sinotti (2004). Moreover, for those who seek recent contributions about the mathematical framework of equilibrium existence and algorithms to find them, Bigi et al. (2013) provides a good review on the subject.

In this paper, our aim is to provide a theoretical criticism of some Nash equilibria in noncooperative games where players have a strict preference for one of the pure strategies of the other players. There are many games in applied and theoretical literature as, for example, the Stag-Hunt game, in which players have a strict preference for one of the other player's pure strategies. Even in such games, there are some Nash equilibria that do not recommend or predict that players should or will collaborate. Thus, we analyze when doing unto others as you would have them do unto you could be considered a reasonable behavior in order to criticize some Nash equilibria.

At this point, it is worth pointing out that differently from the existent arguments against some Nash equilibria, the present one is neither based on a new refinement of the Nash equilibrium concept nor on a new equilibrium selection concept. It is not a refinement because in some games, players do not have a preference for one of the other players' pure strategies regardless of their own actions. And it is not an equilibrium selection because even when players in the game do have preference for one pure strategy of their opponents our argumentation just classifies some equilibria as unreasonable but does not advocate that any other specific equilibrium should be selected. The remaining sections of this paper are organized as follows: in Section 2, we make a brief review about Nash equilibrium and its main criticisms; in Section 3, we introduce the concepts of collaborative dominance and collaborative equilibrium, discussing the rationality principles and the efficiency property of such equilibrium; in Section 4, we present some results about collaborative dominance in two-player zero-sum games; and, in Section 5, we conclude with final remarks.

\section{BACKGROUND}

Roughly speaking, Game Theory is a branch of mathematics that study conflict and cooperation between subjects when the actions taken by each one of them influence their final outcome in the strategic situation. A strategic situation (or simply a game) is composed of three main parts: the players, the strategies available for each player and, for each player, a function (utility function) that for every possible scenario, that is composed of one action for each player in the game, gives a real number that represents the players' preferences (the higher such number is, the more preferred is the consequence induced by the given scenario).

Formally, a strategic or normal form game $G$ is represented by $G=\left(N,\left(S_{i}\right)_{i \in N},\left(U_{i}\right)_{i \in N}\right)$, where $N=\{1, \ldots, n\}$ is a finite set of players, $S_{i}$ is a finite set of (pure) strategies available 
for player $i \in N$, and $U_{i}: \times_{i \in N} S_{i} \rightarrow \mathbb{R}$ is the utility function of player $i \in N$. A strategy profile, $s=\times_{i \in N} s_{i}$, where $s_{i} \in S_{i}$, is a collection of pure strategies, one for each player in $G$, and $S=\times_{i \in N} S_{i}$ is the set of all pure strategy profiles in the game. In order to simplify notation, it is standard to assume that $S_{-k}=\times_{i \in N \backslash\{k\}} S_{i}$ is the set of all strategies profiles considering all players in $G$ but $k$. A mixed strategy for player $k$ is a probability distribution over his pure strategies. Let $\Delta\left(S_{k}\right)$ be the set of all mixed strategies of player $k$, where $\sigma_{k}\left(s_{k}\right)$ indicates the probability that $k$ gives to his pure strategy $s_{k}$ when implementing the mixed strategy $\sigma_{k} \in \Delta\left(S_{k}\right)$. Let $U_{i}(\sigma)$ be player i's expected utility when the mixed strategy profile $\sigma$ is implemented, i.e.

$$
U_{i}(\sigma)=\sum_{s \in S}\left(\prod_{j \in N} \sigma_{j}\left(s_{j}\right)\right) U_{i}(s) .
$$

Let $\left(\sigma_{-k}, \tau_{k}\right)$ be the mixed strategy profile where player $k$ plays according to the mixed strategy $\tau_{k}$ and the other players act according to the strategies specified in $\sigma$. Thus,

$$
U_{i}\left(\sigma_{-i}, \tau_{i}\right)=\sum_{s \in S}\left(\prod_{j \in N \backslash\{i\}} \sigma_{j}\left(s_{j}\right)\right) \tau_{i}\left(s_{i}\right) U_{i}(s) .
$$

We then have that a strategy profile $\sigma=\left(\times_{i \in N} \sigma_{i}\right)$ is a Nash equilibrium of $G$ if, and only if: $U_{i}(\sigma) \geq U_{i}\left(\sigma_{-i}, \tau_{i}\right), \forall i \in N, \forall \tau_{i} \in \Delta\left(S_{i}\right)$. In words, a mixed strategy profile is a Nash equilibrium if no player, acting individually, is able to improve his expected payoff by deviating from the strategy prescribed by the equilibrium.

At this point, it is worth pointing out that Nash equilibrium can be interpreted in many different ways. Nash originally provided two main interpretations of his equilibrium concept: the rational and the evolutionary. Besides Nash, other renowned authors such as Harsanyi (1973) and Aumann (1987) also provided their own view on the subject. However, there is no consensus regarding which of the interpretations is the most appropriate. An interesting discussion about the Nash equilibrium's interpretations can be seen in Osborne \& Rubinstein (1994). In this book, it is possible to see the authors' disagreement on some points. In what follows, we adopt the traditional rational interpretation to develop our argument, i.e. we study one-shot games in which players have some probabilistic mechanism available that enables them to use a mixed strategy.

Almost as well known as Nash equilibrium are its existing criticisms which spread themselves into many different directions, especially regarding mixed equilibrium. Now we briefly present some of them. In the core of these discussions, Nash equilibrium refinements seek (by means of new equilibrium concepts) to eliminate implausible equilibrium points from the original set of equilibria (as, for example, equilibrium profiles composed of weakly dominated strategies). In this literature, we highlight the works of Selten (1975), Myerson (1978) and Kalai \& Samet (1984). Following a different direction of the refinements, Aumann (1974) creates the concept of correlated equilibrium that seeks to increase the set of equilibria adding efficient points to the original set of equilibria (assuming the existence of an impartial mediator).

The other criticisms to the Nash equilibrium concept are mainly focused on the mixed equilibrium (hereafter, mixed means mixed in the non-degenerate sense, i.e. not pure). In this context, we start with the instability argument from Harsanyi (1973) and Harsanyi \& Selten (1988). 
In a mixed strategy Nash equilibrium, players are indifferent among their pure strategies that receive positive probability according to the mixed strategy, i.e. among the pure strategies in the mixed strategy's support. In fact, as the players are indifferent among these pure strategies, any probability distribution over them will give them the same expected utility. Therefore, because there are infinitely many strategies that act as best response to the opponent's mixed strategy, the mixed Nash equilibrium is classified as unstable.

Finally, Aumann \& Maschler (1972) inquired why players should act according to the mixed equilibrium in games where they can guarantee the same expected utility by playing their maximin strategies regardless of the other players' actions. Even though these authors presented an interesting argument against the mixed equilibrium, they did not present a convincing solution on how players should behave in a game, since the maximin strategies of the players may not be a best response to each other. Further discussions about this subject can be found in Pruzhansky (2011).

\section{DOING UNTO OTHERS AS YOU WOULD HAVE THEM DO UNTO YOU}

Consider the games shown in Table 1. First, note that in all of them both players would prefer that the other choose one of the strategies, regardless of which strategy they intend to play. Thus, one may wonder what would lead players not to collaborate in these games. Let us formalize this idea in order to answer this question, defining the following concept.

Table 1

\begin{tabular}{|c|c|c|c|c|c|c|c|}
\hline & \multicolumn{3}{|c|}{ Player 2} & & \multicolumn{3}{|c|}{ Player 2} \\
\hline \multirow{3}{*}{ Player 1} & & $W$ & $Z$ & \multirow{3}{*}{ Player 1} & & $W$ & $Z$ \\
\hline & $X$ & $(3,3)$ & $(0,2)$ & & $X$ & $(0,0)$ & $(3,1)$ \\
\hline & $Y$ & $(2,0)$ & $(1,1)$ & & $Y$ & $(1,3)$ & $(2,2)$ \\
\hline \multicolumn{4}{|c|}{ (i) } & \multicolumn{4}{|c|}{ (ii) } \\
\hline & \multicolumn{3}{|c|}{ Player 2} & & \multicolumn{3}{|c|}{ Player 2} \\
\hline \multirow{3}{*}{ Player 1} & & $\mathrm{~W}$ & Z & \multirow{3}{*}{ Player 1} & & $\mathrm{~W}$ & Z \\
\hline & $X$ & $(1,1)$ & $(0,1)$ & & $X$ & $(3,0)$ & $(0,1)$ \\
\hline & $Y$ & $(1,0)$ & $(0,0)$ & & $Y$ & $(2,3)$ & $(1,2)$ \\
\hline \multicolumn{4}{|c|}{ (iii) } & \multicolumn{4}{|c|}{ (iv) } \\
\hline
\end{tabular}

\section{Weak Collaborative Dominance (or non-strict)}

For any normal form game $\Gamma=\left(N,\left(S_{i}\right)_{i \in N},\left(U_{i}\right)_{i \in N}\right)$, for any player $i \in N$ and for any pure strategy $s_{i} \in S_{i}, s_{i}$ is said weakly collaboratively dominated for player $j \in N$, with $j \neq i$, if exist any mixed strategy $\sigma_{i} \in \Delta\left(S_{i}\right)$ such that

$$
U_{j}\left(s_{i}, s_{-i}\right) \leq \sum_{s_{i}^{\prime} \in S_{i}} \sigma_{i}\left(s_{i}^{\prime}\right) U_{j}\left(s_{i}^{\prime}, s_{-i}\right), \forall s_{-i} \in S_{-i},
$$

with at least one strict inequality. In this case, we say that $\sigma_{i}$ collaboratively dominates $s_{i}$ for player $j$. 
For a strong version of the collaborative dominance definition made above, we should simply use a strict inequality signal $(<)$. Furthermore, by simplicity, in a two-player game, when player $i$ has a collaboratively dominant strategy for player $j$, we say that player $i$ has a collaboratively dominant strategy. Thus, based on the concept of collaborative dominance, we can reformulate the problem presented in the beginning of this section in a more precise way: as long as there is a pure strategy $s_{i}$ of player $i$ that collaboratively dominates all of his other strategies for player $j$ and vice-versa, why would players choose not to collaborate? A possible explanation for players' lack of collaboration is supported by stability purposes, as shown next.

Our aim is to identify which Nash equilibria are unreasonable in games with collaborative dominant strategies. For this purpose, let us analyze in more detail the games shown in Table 1. In all of them, both players have a collaboratively dominant strategy. The question is what happens if each player decides to do unto others as he would have them do unto him? (This idea is known as Luke's Golden Rule (Luke, 6:31)). Or, using the above terminology, what happens if both players choose their collaboratively dominant strategy?

If both choose the collaboratively dominant strategy, then they will achieve a Nash equilibrium in Games (i) and (iii) which is efficient and, therefore, provides an expected utility higher than (or at least equal to) the expected utility of any other Nash equilibria in the respective game for both players. This fact happens because the collaboratively dominant strategies of the players are a best response to each other. However, in Games (ii) and (iv) the pair of collaboratively dominant strategies leads the players to achieve points in the payoff matrix that do not correspond to a Nash equilibrium, i.e. unstable points. This fact happens because, for at least one player, his collaboratively dominant strategy is not a best response to the other player's collaboratively dominant strategy. If a collaborative strategy of player $i$ is not a best response to player $j$ 's collaborative strategy, but the inverse is true, then we say that the collaborative profile is unstable for player $i$. For this reason, we realize that the concept of collaborative dominance is not sufficient to ensure that collaboration will occur. In fact, in Game (iv), the mixed equilibrium is the unique stable strategy profile. Thus, we argue that collaboration (or any other strategy) is a reasonable option if it is part of a Nash equilibrium. Therefore, we define:

\section{Stable Collaborative Dominance (collaborative equilibrium)}

For a given normal form game $\Gamma=\left(N,\left(S_{i}\right)_{i \in N},\left(U_{i}\right)_{i \in N}\right)$, if $\left(\times s_{i \in N}^{*}\right)$ is such that, for all $i \in N$ and for all $s_{i} \in S_{i}, s_{i}$ is weakly collaboratively dominated by the pure strategy $s_{i}^{*}$, for all players $j \neq i$, then, we say that the strategy profile $\left(\times s_{i \in N}^{*}\right)$ is collaboratively stable or a collaborative equilibrium if it is a pure Nash equilibrium, i.e. a strategy profile such that any player that deviates unilaterally from it is not in a better situation.

The next theorem shows that the collaborative equilibrium is efficient.

Theorem 1. Let $\Gamma$ be a game in strategic form. If $\left(\times s_{i \in N}^{*}\right)$ is a collaborative equilibrium, then, for all player $i \in N, U_{i}\left(\times s_{i \in N}^{*}\right)$ is the highest possible utility for player $i$ in $\Gamma$. 
Proof. Assume that $\left(\times s_{i \in N}^{*}\right)$ is a collaborative equilibrium. Thus, by the idea of stability we have: $U_{i}\left(s_{i}^{*}, s_{-i}^{*}\right) \geq U_{i}\left(s_{i}, s_{-i}^{*}\right), \forall s_{i} \in S_{i}$ and $\forall i \in N$. In turn, applying the concept of collaborative dominance for a fixed $s_{j}^{*}$, where $j \neq i$, we have: $U_{i}\left(s_{i}, s_{-i}^{*}\right) \geq U_{i}\left(s_{i}, s_{-i}\right), \forall s_{i} \in S_{i}$, $\forall s_{-i} \in S_{-i}$ and $\forall i \in N$. Therefore, $U_{i}\left(\times s_{i \in N}^{*}\right)$ is higher than or equal to any other utility that player $i$ can achieve in game $\Gamma$. This conclusion applies $\forall i \in N$, ending the proof.

Intuitively, Theorem 1 provides us with an important result: if a collaborative equilibrium exists in a given strategic form game, then no player can get a better result than what he would get if the collaborative equilibrium is played. Note that this is stronger than the usual Pareto efficiency property since it is not only the case that players cannot improve the collaborative equilibrium payoffs without harming another player, but it is the case that every player is getting his favorite outcome in the game. This fact happens because the concept of collaborative equilibrium (when applied) makes use of two principles of rationality: the selfish and the altruistic. The selfish rationality refers to the traditional own utility maximization idea; and the altruistic rationality, in the opposite sense, refers to the maximization of others' utility. Thus, by joining these two ideas, it is possible to ensure that the best outcomes are achieved, what is impossible by applying only one of these principles.

It is important to highlight that for a collaborative equilibrium to be achieved, players do not need to maximize their opponents' utility, it is only necessary that they maximize their own utility and that they believe that the collaborative profile will be played (the same assumptions are necessary for any Nash equilibrium to be achieved). Thus, the fact that other players' utilities are maximized in a collaborative equilibrium is an additional feature that may turn such equilibrium focal. However, this focal property is lost if players are, for example, focusing on security. Hereafter, we refer to a secure equilibrium as one composed of maximin strategies. Game $(i)$, in Table 1, known in the literature as the name Stag-Hunt game, is an example of this efficiencyrisk dilemma. Even though, there is a collaborative equilibrium $(X, W)$, which is efficient by Theorem 1, there is another equilibrium $(Y, Z)$, which is secure. However, note that the mixed equilibrium of this game does not have any of these properties. Thus, besides being stable, players have no other rational reason to play according to the mixed Nash equilibrium in this game. Therefore, in games with a collaborative equilibrium (like Game $(i)$ ), we argue that the only reason for not playing according to such equilibrium is if players are seeking for security, in which case they should play a secure equilibrium. Thus, we identify the mixed equilibrium of Game $(i)$ as unreasonable, because, in such game, it is neither efficient nor secure. Moreover, if players are expecting that other will play according to the mixed equilibrium, then any strategy (including the collaborative or the secure) would be a best response.

Now, we are able to identify what are the Nash equilibria that should be considered unreasonable in a strategic analysis. In a finite normal form game, if a collaborative equilibrium exists, then any equilibrium that is not collaborative or secure should be considered unreasonable. Moreover, it is important to highlight that if the game does not have a collaborative equilibrium, then we do not have any new argument against any Nash equilibrium of the game. 
Again, we do not advocate that players should choose the collaborative equilibrium, which is efficient; they can adopt, for example, another pure or mixed equilibrium with security properties. To illustrate what are the Nash equilibria that we classify as unreasonable let us look at the game described in Table 2.

Table 2

\begin{tabular}{|c|c|c|c|c|c|}
\hline \multirow{5}{*}{ Player 1 } & \multicolumn{5}{|c|}{ Player 2 } \\
\cline { 2 - 6 } & & $A$ & $B$ & $C$ & $D$ \\
\cline { 2 - 6 } & $A$ & $(3,3)$ & $(0,2)$ & $(0,2)$ & $(1,1)$ \\
& $B$ & $(2,0)$ & $(1,1)$ & $(0,0)$ & $(0,0)$ \\
& $C$ & $(2,0)$ & $(0,0)$ & $(1,1)$ & $(0,0)$ \\
& $D$ & $(1,1)$ & $(0,0)$ & $(0,0)$ & $(0,0)$ \\
\hline
\end{tabular}

In such game, strategy $\mathrm{A}$ for both players is collaboratively dominant, and $(A, A)$ is a collaborative equilibrium. Therefore, the game satisfies the requirements for our critique. This game has seven equilibria, namely: $(A, A),(B, B),(C, C),[(1 / 2,1 / 2,0,0),(1 / 2,1 / 2,0,0)]$, $[(1 / 2,0,1 / 2,0),(1 / 2,0,1 / 2,0)],[(0,1 / 2,1 / 2,0),(0,1 / 2,1 / 2,0)]$ and $[(1 / 3,1 / 3,1 / 3,0)$, $(1 / 3,1 / 3,1 / 3,0)]$, in which the last one is a secure equilibrium (because it is composed of maximin strategies). Thus, in our approach, the game in Table 2 has two reasonable equilibria $(A, A)$ and $[(1 / 3,1 / 3,1 / 3,0),(1 / 3,1 / 3,1 / 3,0)]$, because the remaining equilibria do not provide any other advantage for the players and, therefore, playing any one of them could be considered as an unreasonable behavior. So our argumentation excludes these five equilibria from the hall of reasonable equilibria.

\section{COLLABORATIVE DOMINANCE IN TWO-PLAYER ZERO-SUM GAMES}

Based on the concept of collaborative equilibrium and on our early conclusion, we can still point out that two-player zero-sum games do not have collaborative equilibrium. This result is quite intuitive, since zero-sum games have a competitive nature and are characterized by the conflict of interests between the players.

Corollary 2. A two-player zero-sum game does not have a collaborative equilibrium.

Proof. First note that if all players' payoffs are equal to zero, then the game does not have a collaborative equilibrium. Moreover, by Theorem 1, if a collaborative equilibrium exists, then all players get their highest available utilities in the game. Thus, in a two-player zero-sum this cannot happen because if one player gets the highest utility the other one will get the lowest.

The next theorem describes the relation between the concept of collaborative dominance and the standard notion of dominance in zero-sum games.

Theorem 3. In a two-player zero-sum game, as illustrated in Table 3, if $\alpha_{i}$ is strongly (resp. weakly) collaboratively dominant with respect to $\alpha_{j}$ for the column player, then $\alpha_{j}$ will be strongly (resp. weakly) dominant with respect to $\alpha_{i}$. 
Table 3

\begin{tabular}{|c|c|c|c|c|}
\hline \multirow{4}{*}{ Player 1 } & \multicolumn{4}{|c|}{ Player 2 } \\
\cline { 2 - 5 } & & $\beta_{1}$ & $\ldots$ & $\beta_{m}$ \\
\cline { 2 - 5 } & $\alpha_{1}$ & $\left(a_{1,1},-a_{1,1}\right)$ & $\ldots$ & $\left(a_{1, m},-a_{1, m}\right)$ \\
\cline { 2 - 5 } & $\vdots$ & $\vdots$ & $\ddots$ & $\vdots$ \\
\cline { 2 - 5 } & $\alpha_{n}$ & $\left(a_{n, 1},-a_{n, 1}\right)$ & $\ldots$ & $\left(a_{n, m},-a_{n, m}\right)$ \\
\hline
\end{tabular}

Proof. Admit Table 3. Suppose, for simplicity, that the strategy $\alpha_{1}$ of Player 1 is strongly (resp. weakly) collaboratively dominant with respect to strategy $\alpha_{n}$ for Player 2, implying that $-a_{1, j}>-a_{n, j}$, for $j=1, \ldots, m$ (resp. $-a_{1, j} \geq-a_{n, j}$, for $j=1, \ldots, m$, with at least one strict inequality). But $-a_{1, j}>-a_{n, j}$ if, and only if, $a_{1, j}<a_{n, j}$ (resp. $-a_{1, j} \geq-a_{n, j}$ if, and only if, $a_{1, j} \leq a_{n, j}$ ), then this leads us to conclude that the strategy $\alpha_{1}$ is strongly (resp. weakly) dominated by $\alpha_{n}$. A similar conclusion is also valid for Player 2.

Corollary 4. If in a two-player zero-sum game, as illustrated in Table 3, the strategy $\alpha_{i}$ of Player 1 is collaboratively dominated by all other strategies $\alpha_{j}$ for Player 2 and if the strategy $\beta_{j}$ of Player 2 is collaboratively dominated by all other strategies $\beta_{k}$ for Player 1 , then the pair $\left(\alpha_{i}, \beta_{j}\right)$ is a Nash equilibrium of the game. Furthermore, if the collaborative dominance is strong, then the equilibrium is unique.

Proof. By Theorem 3, we know that if a strategy is strongly (resp. weakly) collaboratively dominated by another strategy, then it is strongly (resp. weakly) dominant in relation to this strategy. Therefore, given the profile $\left(\alpha_{i}, \beta_{j}\right)$, no player has an incentive to deviate from this profile, so it is a Nash equilibrium. If dominance is strong, then we have that the profile $\left(\alpha_{i}, \beta_{j}\right)$ is the only one that survives the process of elimination of strongly dominated strategies. Therefore, the equilibrium is unique.

\section{FINAL REMARKS}

As it is the most important solution concept in game theory, Nash equilibrium is constantly kept under review. In this article, we analyze how reasonable it is to play according to some Nash equilibria if players have a preference for one of their opponents' strategies. In order to formalize our argument, we propose the concepts of collaborative dominance and collaborative equilibrium (which is a strict pure Nash equilibrium). First we proved that, when the collaborative equilibrium exists it is efficient, i.e. it is impossible for any player to achieve a better outcome than that provided by the collaborative equilibrium. This first result is important for those who study social welfare or advocate collaborative behavior; because it provides a reason why collaboration (when a collaborative equilibrium exists) could become a focal behavior, without making any other assumption. Experimentalists can also benefit from the concepts proposed here in order to justify particular behaviors obtained in their studies. 
We saw that a reason for players not to collaborate occurs when they are focusing in security instead of efficiency and, therefore, they shall play a secure equilibrium. This argument allows us to reduce the hall of reasonable equilibria for games where a collaborative equilibrium exists. Therefore, this paper brings new theoretical contributions to the literature on criticisms of Nash equilibrium in the sense that it proposes an argument that is neither based on a new refinement nor on a new equilibrium selection concept, making it less restrictive and simple (when applied), without losing the mathematical formalism required by the subject.

Finally, we proved that two-player zero-sum games do not have collaborative equilibrium, and that if there exists a strategy profile composed only of collaboratively dominated actions, then it is a Nash equilibrium of the game. Thus, our approach allows a new way to highlight the competitive nature of zero-sum games, which (in such cases) prevent us to do unto others as we would have them do unto us.

\section{ACKNOWLEDGMENTS}

The authors would like to thank Joseph Halpern, André Leite Wanderley and anonymous referees for helpful advices. The authors also would like to thank the Conselho Nacional de Desenvolvimento Científico e Tecnológico (CNPQ) for financial support.

\section{REFERENCES}

[1] Aumann RJ. 1974. Subjectivity and Correlation in Randomized Strategies. Journal of Mathematical Economics, 1: 67-96.

[2] Aumann RJ. 1987. Correlated Equilibrium as an Expression of Bayesian Rationality. Econometrica, 55: $1-18$.

[3] Aumann RJ \& Maschler M. 1972. Some thoughts on the minimax principle. Management Science, 18: 54-63.

[4] Bigi G ET AL. 2013. Existence and solution methods for equilibria. European Journal of Operational Research, 227: 1-11.

[5] Harsanyi JC. 1973. Games with Randomly Disturbed Payoffs: A New Rationale for Mixed Strategy Equilibrium Points. International Journal of Game Theory, 2: 1-23.

[6] Harsanyi JC \& Selten R. 1988. A General Theory of Equilibrium Selection in Games. London: MIT Press.

[7] Kalai E \& Samet D. 1984. Persistente Equilibria. International Journal of Game Theory, 13: $129-144$.

[8] KUhn HW \& TUCKER AW. 1958. John von Neumann's work in the theory of games and mathematical economics. Bulletin of the American Mathematical Society, 64: 100-122.

[9] Leonard RJ. 1995. From Parlor Games to Social Science: Von Neumann, Morgenstern, and the Creation of Game Theory, 1928-1994. Journal of Economic Literature, 33: 730-761.

[10] Machado HV \& Sinotti RL. 2004. Conflito em redes: origens e destinos cotados. Pesquisa Operacional, 24: 163-180. 
[11] Migdalas A. 2002. Applications of Game Theory in Finance and Managerial Accounting. Operational Research, 2: 209-2041.

[12] Myerson RB. 1978. Refinements of the Nash Equilibrium Concept. International Journal of Game Theory, 7: 73-80.

[13] NASAR S. 1998. A Beautiful Mind: A Biography of John Forbes Nash, Jr. New York: Simon.

[14] NASH JF. 1951. Non-Cooperative Games. The Annals of Mathematics, 54: 286-295.

[15] Osborne MJ \& Rubinstein A. 1994. A Course in Game Theory. Cambridge Mass: MIT Press.

[16] Poundstone W. 1992. Prisoner's Dilemma: John von Neumann, Game Theory, and the Puzzle of the Bomb. Oxford: Oxford University Press.

[17] PRUZHANSKY V. 2011. Some interesting properties of maximin strategies. International Journal of Game Theory, 40: 351-365.

[18] SELten R. 1975. A reexamination of the perfectness concept for equilibrium points in extensive games. International Journal of Game Theory, 4: 25-55.

[19] WANG Q \& PARLAR M. 1989. Static game theory models and their applications in management science. European Journal of Operational Research, 42: 1-21. 